\title{
Diminished mixed lymphocyte reaction in ankylosing spondylitis, relatives, and normal individuals all with HL-A 27
}

\author{
B. NIKBIN, D. A. BREWERTON, D. C. O. JAMES, AND J. R. HOBBS \\ From the Departments of Chemical Pathology and Rheumatology, and Tissue Typing Laboratory, \\ Westminster Hospital, London
}

\begin{abstract}
Nikbin, B., Brewerton, D. A., James, D. C. O., and Hobbs, J. R. (1976). Annals of the Rheumatic Diseases, 35, 37-39. Diminished mixed lymphocyte reaction in ankylosing spondylitis, relatives, and normal individuals all with HL-A 27. The mixed lymphocyte reaction against a pool of blocked lymphocytes was studied in individuals with and without HL-A 27, including controls, spouses, parents, sibs, and offspring, and patients with ankylosing spondylitis. In controls without HL-A 27 the mean increment of counts perminute was 46630 compared with 29860 in asymptomatic controls and relatives with HL-A 27 and 26277 in spondylitic patients with HL-A 27. The mean response was also reduced to 34902 in sibs and offspring without HL-A 27, and to 20916 in three patients with spondylitis in the absence of HL-A 27.
\end{abstract}

In a pilot investigation of lymphocyte function in ankylosing spondylitis the mixed lymphocyte reaction (MLR) was reduced in patients with ankylosing spondylitis and in their first-degree relatives with HL-A 27, suggesting the possibility of an inherited defect of cell-mediated immunity (Nikbin, Brewerton, Byrom, James, Malka, McLeod, Slater, Warren, and Hobbs, 1975). Therefore the investigation has been repeated and extended, including patients and relatives without HL-A 27. As the initial investigation had shown no difference in the pattern of results using autologous or $\mathrm{AB}$ serum, it was decided to use autologous serum only in the second study.

\section{Patients and methods}

Twenty-eight patients had ankylosing spondylitis (New York criteria): 25 had HL-A 27 and three did not. None had a history of urethritis or evidence of Reiter's disease, inflammatory bowel disease, or psoriasis. All were known to be in a quiescent phase, the majority requiring no drugs, not even analgesics. The patients were contacted in advance and agreed to take no drugs for 3 weeks before attending a special clinic for investigation, at which a rheumatologist (D.A.B.) confirmed verbally that no drugs had been taken. Fifty-threefirst-degree relatives of patients with ankylosing spondylitis were selected from those who had assisted in previous studies, excluding all who had had rheumatic symptoms, uveitis, or evidence of other relevant diseases. Of these asymptomatic relatives, eight parents, four sibs, and nine offspring had HL-A 27, while eleven parents, thirteen sibs, and eight offspring did not. Fourteen symptom-free spouse controls were selected, excluding those who themselves had a family history or offspring with rheumatic diseases; two of the fourteen had HL-A 27. At investigation all relatives and spouses were interviewed by the rheumatologist to confirm that they fulfilled all the criteria and had taken no drugs. The controls were blood donors and hospital staff previously tissue-typed; sixteen had HL-A 27 and 22 did not. All were symptom free, were taking no drugs, and had no family history of spondylitis. The blood was delivered to the laboratory in mixed, unidentified batches, and to limit technical variation all investigations were completed within an 8-week period. HL-A antigens were determined by a modified, two-stage lymphocytotoxicity micromethod (Terasaki and McClelland, 1964). The MLR was standardized for use in the flat-bottomed wells of Cook microplates, with harvesting using a Mash II (Dynatech Ltd.). Lymphocytes were separated using Lymphoprep. Since a re-evaluation of the method had indicated that this was the most convenient and reliable immunogen, the stimulating lymphocytes were provided by a fresh pool of five random donors, and they were blocked with mitomycin-C. It had also been established that the best results were obtained with an equal number of responding and stimulating cells (100000 of each) in medium TC 199 to which was added 
$10 \%$ autologous serum. Incubation was for 120 hours at $37^{\circ} \mathrm{C}$. ${ }^{3} \mathrm{H}$-thymidine was added to a concentration of $10 \mu \mathrm{Ci} / \mathrm{ml}$, with harvesting 15 hours later. Testing this method in 29 healthy young adults the results fitted a Gaussian distribution better than the log-normal distributions observed against phytohaemaglutinin and Candida immunogen and previously in the MLR. In the present study the results resembled a Gaussian distribution, but it was decided to use a distribution-free test (Wilcoxon two-tailed test for unpaired observations) to assess the significance of differences in mean values between the clinical groups investigated.

\section{Results}

The results are expressed in increments of counts per minute, taking the mean of each clinical group (Fig. 1). In controls without HL-A 27 (representing approximately $93 \%$ of the population) the mean was 46630. Compared with this the mean in patients with ankylosing spondylitis and HL-A 27 was 26277 $(P<0.005)$. There were only three patients without HL-A 27 with a mean of 20916 (P $<0.02)$. In asymptomatic individuals with HL-A 27 the means were: controls $28441(\mathrm{P}<0.02)$, parents 33123 (not significant), and sibs and offspring $28384(\mathrm{P}<0.002)$. These results in asymptomatic individuals with $\mathrm{HL}-\mathrm{A}$ 27 did not differ significantly from the mean in spondylitic patients with $\mathrm{HL}-\mathrm{A} 27$. In asymptomatic individuals without HL-A 27 the means were: spouses 39438 (not significant), parents 41175 (not significant), and sibs and offspring 34902 ( $P<0.05)$. In the latter group the mean in eight offspring was 34424

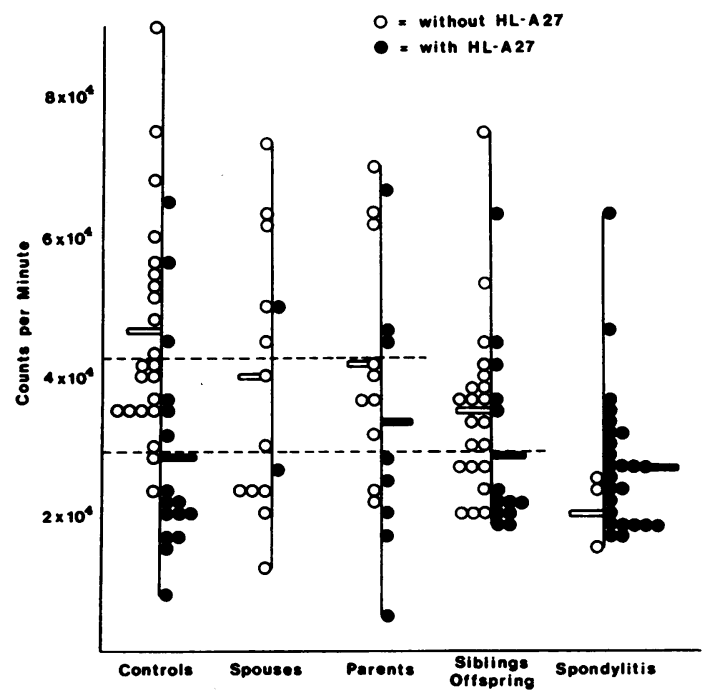

FIG. 1 Results of the mixed lymphocyte reaction expressed as increments of counts per minute. The dotted lines represent the mean of controls, spouses, and parents without HL-A 27 (42933) and of controls, spouses, parents, sibs, and offspring with HL-A 27 (29860) and in thirteen sibs 35208 . The eleven parents without HL-A 27 had a mean of 41175 compared with 33123 in the eight parents with HL-A 27 (not significant). Taking all asymptomatic groups together there were 39 individuals with HL-A 27 and a mean of 29860 compared with 66 without HL-A 27, who had a mean of $40682(P<0.002)$. One family is illustrated in Fig. 2.

\section{Discussion}

The diminished MLR in patients with ankylosing spondylitis has been confirmed. Although patients were selected whose disease was quiescent and who were not taking drugs, this finding alone does not indicate whether the diminished MLR represents an inherited defect or a result of the disease. Of more importance are the diminished responses in all asymptomatic groups with HL-A 27 . These suggest that the diminished responses in the MLR may represent a defect of cell-mediated immunity inherited with HL-A 27, and it appears that this association may be widespread in the population rather than only in patients and their relatives. Even in the absence of HL-A 27 there may be diminished responses in patients with spondylitis and (to a lesser extent) in sibs and offspring, so that the defect may be inherited independently as well as in association with HL-A 27.

It is not known whether HL-A antigens are directly involved in disease processes or whether they denote abnormal immune responses inherited in close association with them (McDevitt and Bodmer, 1974). Similarly, it is not yet clear what importance the diminished MLR may have in susceptibility to disease. In this investigation there were many individuals with both HL-A 27 and low MLR responses who had never suffered any rheumatic symptoms, so that there must be further factors in the aetiology of ankylosing spondylitis. Nevertheless, a defect of cellmediated immunity represented by a diminished

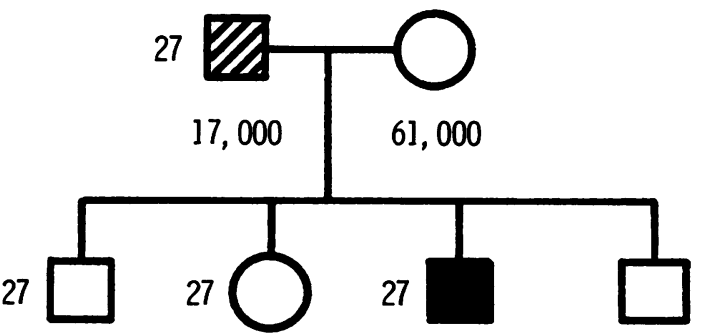
20,000

18,000

37,000

FIG. 2 Results of the mixed lymphocyte reaction in a family. The father with HL-A 27 had uveitis and symptomatic spondylitis with normal $\mathrm{x}$-rays. Three offspring had $H L-A$ 27: two asymptomatic and one with definite ankylosing spondylitis 
MLR response might prove to be more closely related to disease susceptibility than the inheritance of HL-A 27, with which it is associated.

We thank the patients, relatives, and controls for their co-operation. Many colleagues at Westminster gave generous help, particularly Mr. N. A. Byrom and Dr. S. Sengupta. Misses M. Caffrey, P. Zylinski, and C. Cockburn did most of the tissue-typing. Mr. David Brown supervised the computer analysis. Dr. R. M. Mason and Professor H. L. F. Currey of the London Hospital kindly referred two patients without HL-A 27, and Dr. R. Grahame of Guy's Hospital three patients with HL-A 27. Blood from controls with HL-A 27 was supplied by Drs. C. C. Entwistle, W. J. Jenkins, and K. Ll. Rogers, as well as by our own staff and blood donors. The project was financed by a generous grant from the Arthritis and Rheumatism Council.

\section{References}

MCDevitT, H. O., AND Bodmer, W. F. (1974) Lancet, 1, 1269 (HL-A, immune-response genes, and disease)

Nikbin, B., Brewerton, D. A., Byrom, N., James, D. C. O., MAlka, S., McLeod, L., Slater, L., Warren, R. E., AND HoBBS, J. R. (1975) Ann. rheum. Dis., 34, Suppl. 1, 49 (Lymphocyte function in ankylosing spondylitis)

Terasaki, P. I., AND McClelland, J. D. (1964) Nature, 204, 998 (Microdroplet assay of human serum cytotoxins) 